\title{
Qualität und Evaluation - Implizite Wirkungsattribute und methodische Herausforderungen
}

\section{Désirée Donzallaz}

Der Beitrag fokussiert das normative Spannungsfeld von Qualität und Evaluation vor dem Hintergrund bildungspolitischer Steuerungsabsichten und methodischer Wirkungsansprü̈che. Qualität übernimmt im Bildungssystem in der gegenwärtigen Akzentuierung die Funktion einer präskriptiven Kategorie, die sowohl für das Bildungssystem als auch die einzelne Bildungsinstitution handlungsrelevant ist. Als Ziel- und Programmkategorie stützt sich Qualität dabei auf ein normatives Fundament, welches auf einer allgemeinen begrifflichen Ebene zunächst nicht weiter expliziert wird und den Qualitätskonzepten inhärent ist. Am Beispiel des nationalen Projekts "Zertifizierung von berufsbildenden Schulen" wird aufgezeigt, dass ein wenig expliziertes Qualitätsverständnis zu Wirkungseinschätzungen führt, die unspezifisch gehalten sind, von impliziten, kontextimmanenten Faktoren gesteuert werden und nur beschränkt nutzbringende Erkenntnisse über tiefer liegende Wirkungsmechanismen sowie Gestaltungsmöglichkeiten liefern.

\section{Grundannahmen}

Qualität und Evaluation sind aus dem Bildungssystem nicht mehr wegzudenken. Auf der Grundlage des neuen Modells zur Steuerung des Bildungssystems mittels Output- und Wettbewerbssteuerung (Bellmann, 2006, S. 489) bei gleichzeitiger internationaler Neuausrichtung der Bildungspolitik (Criblez \& Osterwalder, 2004, S. 5) übernehmen Qualität und Evaluation als zentrale Steuerelemente eine gewichtige Funktion. Wenn Qualität mit Exzellenz gleichgesetzt wird und die Evaluation im Kleide internationaler Schulleistungsvergleichsstudien wie TIMMS und PISA dies nachweisen soll, dann verweist dies auf eine funktionale und normative Komponente, die ihre Wirkungskraft erst im spezifischen Kontext entfaltet. Qualität und Evaluation sind als bildungspolitisch besetzte Konzepte wandlungsfähig: Welches Qualitätsverständnis sich mit welchen Wirkungsabsichten in der Tiefenstruktur verbirgt und durch die Evaluation verarbeitet wird, kann nur durch Betrachtung des Rezeptions- und Bewertungspro- 
zesses von Qualitätskonzepten aufgedeckt werden.

Vorliegender Beitrag thematisiert das Verhältnis von Qualität und Evaluation in Form des konkreten Bestrebens, Qualitätskonzepte ${ }^{1} \mathrm{zu}$ evaluieren und Wirkungsnachweise aufzuzeigen. Anhand des vom Bundesamt für Berufsbildung und Technologie BBT 1995 initiierten und 2003 abgeschlossenen Projekts «Zertifizierung von berufsbildenden Schulen» wird das Verhältnis zwischen pädagogischer Rezeption und bildungspolitischer Evaluation aufgezeigt. Dieses Fallbeispiel unterstreicht die These des Beitrags, dass die Wirkungsattribute zwischen der Rezeption und der Evaluation differieren und von impliziten, kontextimmanenten Faktoren gesteuert werden. Während die Rezeption von impliziten und unterschiedlichen Wirkungsannahmen geprägt ist, bewegt sich die Evaluation mit der Wirkungsfeststellung auf einer Ebene, die dem Qualitätskonzept als nicht näher spezifizierte Programmkategorie eine hohe Wirkungskraft beimisst. Dies soll mit der nachfolgenden Diskussion aufgezeigt werden.

\section{Qualität als präskriptive Ziel- und Programm- kategorie}

Die Konfrontation und Auseinandersetzung mit divergierenden, zuweilen polemisierenden Haltungen und Aussagen ist unumgängliche Basis im Zusammenhang mit der wissenschaftlichen Beschäftigung mit Qualität im Bildungssystem. Das Spektrum reicht dabei von der Betonung der Normativität, Relativität und Unmöglichkeit, Qualität näher definieren zu können, über Bemühungen zur akribischen und ganzheitlichen Beschreibung und Auflistung von Qualität in Merkmalslisten bis hin zu Annahmen, die die Qualität als umsetzbare, objektiv messbare und stabile Grösse propagieren. Trotz unterschiedlicher Positionen und kritischer Einwände wird Qualität vorwiegend mit etwas Gutem, Positivem und Wünschenswertem gleichgesetzt, das sich im Gewand von konkreten Programmen, in Form von Exzellenzwünschen oder in Bemühungen um Bildungsstandards zeigen kann. Qualität wird damit einerseits wandlungsfähig und andererseits automatisch auf eine unterschiedliche normative Grundlage gestellt. Auf einer allgemeinen, begrifflich nicht weiter umschriebenen Ebene scheint ein unausgesprochener Konsens über Qualität als Ziel- und Reformprogrammkategorie zu bestehen, was letztlich eine apodiktische Haltung begünstigt. So hat die Bildungspolitik Qualität längst schon zum gesetzlich verankerten Leitkonzept (neues Berufsbildungsgesetz, 2000; Neuordnung der Bildungsartikel in der Bundesverfassung, 2006) und damit zum verordneten Reformprogramm für die einzelne Schule erklärt. Heid (2000) weist im Zusammenhang mit dem Qualitätsdiskurs jedoch auf die Gefahr hin, dass eine abstrakte Kennzeichnung des Gegenstandes «tendenziell» die Einigkeit über diesen erhöht und gleichzeitig das Risiko birgt, die Unterschiede in den Beurteilungen zu verdecken (S. 45). Ungeachtet dieses Einwands hat das $Q$ als Präfix in vielen Schulreformkonzepten und 
-programmen für die einzelne Bildungsstätte längst den Status eines Kollektivsymbols: Bezogen auf die Interaktion und die Bedeutung der Teams für die Entwicklung und Steuerung der Schule spricht man gerne von Q-Gruppen und QZirkeln; in Q-Konzepten, Q-policies und Q-Handbüchern werden konkrete Q-Massnahmen beschrieben, die in periodischen Q-Evaluationen überprüft und durch Q-Zertifikate formal bestätigt werden. Konsens besteht nicht nur über den Qualitätsbegriff als reformpolitisches Programm, sondern auch darüber, dass Qualität wie selbstverständlich, intern und extern, top down und bottom up gesteuert, evaluiert und scheinbar verbessert wird. Für die Einzelschule aller Bildungsstufen gibt es eine Vielzahl an «How-to-do-Konzepten» («Qualität durch ... Formulierungen», hierzu z.B. Landwehr \& Steiner, 2003, oder Maritz et al., 2006), die präskriptiv Qualität hervorbringen, steuern und verbessern wollen und damit - wenn auch nicht explizit - letztlich auch normativ sind.

Für die wissenschaftliche Beschäftigung mit Qualitätsfragen im Bildungssystem bedeutet dies, dass das normative Fundament im Zusammenhang mit der Qualitätsdiskussion nicht ausgeblendet werden kann und deshalb zwangsläufig selbst Gegenstand der Auseinandersetzung wird. Diese Notwendigkeit wird mit Blick auf die Wirkungsforschung zusätzlich unterstrichen. Die methodischen Herausforderungen der Überprüfung der Wirkungsfrage von Qualitätskonzepten in Bildungsorganisationen liegen darin begründet, dass einfache kausale Zusammenhangshypothesen nicht ausreichend sind, um der Komplexität und Vielschichtigkeit der Wirkungsfrage in Organisationen gerecht zu werden. So werden denn auch das Komplexitätsargument im Sinne von unzählig vielen intervenierenden und nicht zu kontrollierenden Variablen (Helmke \& Weinert, 1997; Hesse \& Wottawa, 1997, zur Problematik des Konzepts der multiplen Determination der Schulleistung) ${ }^{2}$ als auch der Bildungsgegenstand selbst hervorgehoben, um Wirkungsannahmen ungeprüft zu lassen (Scheerens \& Bosker, 1997, zur Problematik der Schuleffektivitätsforschung). Für Heid (2000) ist dieser Unterschied aber nicht nur durch methodische Hürden begründet, sondern in der Tatsache, dass gegenüber den allgemein formulierten Programmentwürfen im konkreten Unterricht unterschiedliche Qualitätsaspekte relevant sind und diese auch subjektiv interpretiert und bewertet werden (S. 46f.). Diese Feststellung kann mit der Betrachtung der Qualitätsfrage anhand der Schule im Systemzusammenhang unterstrichen werden, die aufzeigt, dass es für das Bildungssystem keine Globaltheorie gibt, die einen Gesamtzusammenhang zwischen allen Elementen, d.h. zwischen gesellschaftspolitischen Ansprüchen, der einzelnen Schule, dem Unterricht und dem Individuum herstellt und die Beziehungen aller Elemente und Ebenen enthält (Lersch, 2004, S. 74). Damit wird nachvollziehbar, dass es letztlich keine eindeutigen empirischen Belege über die Wirksamkeit von Qualitätsansprüchen im Bildungssystem geben kann, die in logischer Abfolge die Auswirkungen von präskriptiven Qualitätsprogrammen sowohl auf der Ebene der Einzelschule, des Unterrichts als auch des einzelnen Lernenden nachzuweisen vermögen. ${ }^{3}$ 
An dieser Stelle kann festgehalten werden, dass Qualität im Bildungssystem in der gegenwärtigen Akzentuierung die Funktion einer präskriptiven Kategorie übernimmt und sowohl für das Bildungssystem als auch die einzelne Schule handlungsleitend ist. Als Ziel- und Programmkategorie stützt sich Qualität dabei auf ein normatives Fundament, das auf einer allgemeinen begrifflichen Ebene zunächst nicht weiter expliziert wird und den Qualitätskonzepten inhärent ist. So wird auf den ersten Blick nicht ersichtlich, was genau unter Qualität verstanden, wie diese bewertet und in welchem nachweislichen Begründungsund Wirkungszusammenhang diese letztlich gestellt wird. Für die weitere Auseinandersetzung wird deshalb die Frage nach der Bewertung bzw. der Bestimmung von Qualität im weitesten Sinne relevant.

\section{Qualität als eine im Kontext stehende Bewertungskategorie}

Qualität, gedeutet als «Bewertungsergebnis» über die «Beschaffenheit eines Objektes», entfaltet ihre Bedeutung erst im jeweilig spezifischen Kontext (Heid, 2000, S. 41). Qualität ist in dieser Hinsicht kein stabiles Konstrukt, dem «inhärente Merkmale» ${ }^{4}$ zugrunde liegen. Qualität ist vielmehr das Resultat einer Reihe von Entscheidungen, das von verschiedenen Kriterien beeinflusst wird und sich erst unter Einbeziehung des Kontextes entschlüsseln lässt (S. 41f.). Etwas kann nur dann als gut oder schlecht bezeichnet werden, wenn ein «entscheidungsabhängiges (normatives) Beurteilungs- bzw. Bewertungskriterium» herbeigezogen wird und dieses Kriterium eine "wertende Stellungnahme zu dieser Sache» (Heid, 2000, S. 42) ist. Deshalb plädiert Heid für ein Verfahren, das nicht nur der Bestimmung und Sicherung von Qualität gilt, sondern auch ein Vorgehen enthält, das sich der Entwicklung und Begründung der Bestimmung einschlägiger Kriterien verschreibt. Durch Betonung der Bewertungskomponente bekommt Qualität eine dynamische, handlungsorientierte Komponente im Zusammenhang mit der Erbringung einer Bewertungsleistung, was auch mit Dubs (2003, S. 15) aufgezeigt werden kann, wenn er im Zusammenhang mit der Entstehung von Qualität von einem politischen Aushandlungsprozess spricht. Konkret kann gefragt werden, an welchen Qualitätsverständnissen sich unterschiedliche Interessengruppen bei ihren Bewertungen letztlich orientieren. Harvey und Green (2000) haben eine Klassifikation erstellt, die genau dieser Frage nachgeht und Qualität als von verschiedenen Disziplinen beeinflusstes Konstrukt mit unterschiedlichen Gesichtspunkten deutet. Sie unterscheiden fünf verschiedene Kategorien, mit welchen Qualität im Bildungssystem näher umschrieben werden können: Neben Qualität als dem Aussergewöhnlichen, der Qualität als Perfektion oder Konsistenz, der Qualität als Zweckmässigkeit und der Qualität als adäquatem Gegenwert umschreiben sie Qualität als Transformation (Harvey \& Green, 2000, S. 17ff.). 
Mit der Vorstellung der Qualität als dem Aussergewöhnlichen geht ein Verständnis einher, wonach Qualität etwas Besonderes ist, wovon sich drei verschiedene Varianten unterscheiden lassen: (1) Die Vorstellung von Qualität als herausgehobene Ausnahme, die als traditionelle Vorstellung Exklusivität und Unerreichbarkeit impliziert (Harvey \& Green, 2000, S. 19). (2) Qualität als Exzellenz basierend auf der Annahme, dass Qualität etwas Vortreffliches ist und durch das Einhalten bzw. Übertreffen allerhöchster Standards zustande kommt (Harvey \& Green, 2000, S. 21). (3) Das dritte Qualitätsverständnis beinhaltet die Konformität von Produkten oder Dienstleistungen mit gesetzten (Minimal-) Standards (Harvey \& Green, 2000, S. 20f.).

Mit der Auffassung der Qualität als Perfektion oder Konsistenz verlagert sich die Sichtweise von den Inputs und Outputs auf die Prozesse. Qualität ist dann gegeben, wenn vordefinierte und messbare Spezifikationen im Sinne von Anforderungen an ein Produkt oder eine Dienstleistung kontinuierlich fehlerlos erfüllt und damit zur Perfektion werden (Harvey \& Green, 2000, S. 21f.).

Der Sichtweise der Qualität als Zweckmässigkeit liegt ein funktionales Verständnis zugrunde, wonach Qualität dann vorhanden ist, wenn sie einen bestimmten Zweck erfüllt. Dieser kann sowohl von der Bildungsinstitution selber bestimmt oder aber von verschiedenen Interessengruppen beeinflusst werden (Harvey \& Green 2000, S. 23ff.).

Die Vorstellung von Qualität als adäquatem Gegenwert für das investierte Geld verkörpert eine allgemeine Konsum- und Kundenhaltung, die für wenig Geld einen möglichst hohen Standard erwartet. Die Kopplung von Qualität mit monetären Erwartungen steht unausweichlich in Verbindung mit einem Marktund Konkurrenzmechanismus, der eine höhere Effektivität zur Folge haben soll (Harvey \& Green, 2000, S. 28).

Qualität als Transformationsprozess betont den qualitativen Aspekt und entspricht dem pädagogischen Verständnis des Bildungs- und Erziehungsauftrags einer Bildungsinstitution. Erziehung und Bildung werden nicht als Dienstleistung für den Kunden aufgefasst, sondern bezwecken als originär pädagogische Aufgabe eine kontinuierliche Weiterentwicklung (enhancing) und Ermächtigung (empowerment) der Lernenden in ihrer Rolle als Ko-Produzenten (Harvey \& Green, 2000, S. 31).

Mit diesen Ausführungen wird deutlich, dass die konkreten Qualitätsvorstellungen letztlich unterschiedliche Steuerungs- und Wirkungsannahmen beinhalten, die durchsetzt von politischen, ökonomischen und pädagogischen Motiven die normative Grundlage für die Evaluation bilden. Für die Evaluation ergeben sich daraus zwangsläufig weitreichende Konsequenzen. Die Evaluation, in diesem Beitrag gedeutet als Instrument zur Wirkungsüberprüfung von Qualitätskonzepten, hat im praktischen Vollzug mehrere Herausforderungen anzunehmen: Bei der Erbringung von Wirkungsnachweisen gilt es - was ex ante versäumt wurde -, zu entscheiden, was genau, d.h. welche Vorstellungen, Dimensionen und Kriterien einem Qualitätskonzept zugrunde liegen und wie diese 
zu bewerten sind. Irgendwann muss entschieden werden, ob das Programm erfolgreich und unterstützungswürdig ist. Wie die Evaluation bei der Explizierung der normativen Grundlage schliesslich umgehen kann, ist Gegenstand der folgenden Ausführungen.

\section{Qualität mittels Evaluation bestimmen, überprüfen und Legitimieren}

Im Qualitätsdiskurs taucht Evaluation an verschiedenen Stellen auf: als Instrument zur Legitimation von Qualitätskonzepten, als eigenständiges MonitoringInstrument zur regelmässigen Überprüfung des Zustands der getroffenen Massnahmen im Rahmen von Qualitätssicherungskonzepten oder gar in der Funktion als eigenständiges Qualitätskonzept. Das Verhältnis von Qualität und Evaluation wird in unterschiedlicher methodischer Weise diskutiert; als Problematik im Zusammenhang mit der Operationalisierung des Qualitätsbegriffs (Kromrey, 2004; Stockmann, 2002) sowie als Herausforderung, die es im Zusammenhang mit der Bewertung eines unspezifizierten Gegenstands zu meistern gilt (Heid, 2000; Stockmann, 2002).

Kromrey (2004) bemerkt, dass die «Unbestimmtheit des Begriffs» für die Evaluation ein zentrales theoretisches und methodologisches Problem beinhaltet (S. 240f.). Qualität ist als Konstrukt gerade wegen seiner «Vieldimensionalität» und seiner unterschiedlichen kontextabhängigen Geltung für verschiedene Interessengruppen nicht in einer Weise "empirisch abbildbar», wie es das wissenschaftliche Konzept «Messen» verlangt (Kromrey, 2004, S. 241). Auch Heid (2000) thematisiert das Problem der Messbarkeit von Qualität und verweist auf einen weiteren relevanten Aspekt, nämlich, dass bei der Evaluation die Qualitäts- und Beurteilungskriterien operationalisiert werden müssen und die Evaluation damit auch Auswirkungen auf deren Bestimmung hat (S. 49). Ebenso stellt Stockmann (2002) die Frage nach der Bestimmung des Qualitätsbegriffs und seiner Überprüfbarkeit. Er stellt fest, dass eine «griffige Spezifikation» fehlt und die offenbar auch technischen Normen (wie zum Beispiel die ISO 9000er Normenserien) zugrunde liegenden Überprüfungen mittels objektiver Kriterien einer subjektiven Beurteilung unterliegen (S. 209). Mit diesen Ausführungen wird deutlich, dass die Evaluation von Qualität eine Frage der Messbarkeit und damit verbunden der Operationalisierung des Qualitätsgegenstandes ist. Das normative Problem wird an dieser Stelle noch einmal sehr gewichtig, denn ungeklärt ist, wie die Evaluation damit konkret umgehen kann, welche Vorgehensweise ihr bei der Bewältigung dieser Aufgabe helfen kann.

In der Praxis verpflichtet sich die Evaluation als wertende Methode, valide Informationen zur Verfügung zu stellen, um den Wert eines Gegenstandes abzuwägen (Glossar wirkungsorientierte Evaluation, Beywl, 2004, S. 10). Die Evaluation verschreibt sich damit einem professionellen Vorgehen, das - gestützt auf 
Evaluationsstandards - durch ausreichende Berücksichtigung unterschiedlicher Bedürfnisse und Interessen von potenziellen Nutzern und Nutzerinnen / Stakeholder nützlich und durchführbar ist, ethisch und rechtlich angemessen vorgeht sowie gültige und brauchbare Erkenntnisse ermittelt (Evaluations-Standards der Schweizerischen Evaluationsgesellschaft, 2000; Standards des Joint Committee on Standards for Educational Evaluation, 2000). Eine Orientierung an diesen Standards ermöglicht neben der Herstellung von Transparenz in Bezug auf verschiedene Vorstellungen über die Evaluation und deren Nutzen die Klärung unausgesprochener Wertfundamente sowie die Bestimmung der Bewertungskriterien für die Evaluationsergebnisse. Die Aufgabe, die Wirkungsfrage von Qualitätskonzepten zu beantworten, kann mittels einer wirkungsfeststellenden und wirkungsorientierten Evaluation erfüllt werden. Die Evaluation hat zu belegen, dass empirisch erfasste Ergebnisse auf das Konzept zurückzuführen sind und vom Qualitätskonzept beabsichtigte Wirkungen berücksichtigt werden (Glossar wirkungsorientierte Evaluation, Beywl et al., 2004, S. 45f.). Konkret kann dies mittels Wirkungseinschätzungen, Wirkungsmodellierungen oder Wirkungsidentifizierungen durchgeführt werden (Beywl et al., 2004, S. 44f.). Bezogen auf das methodische Anspruchsniveau zeigt sich, dass Wirkungseinschätzungen mit Hilfe «typischer Evaluationsfragen», wie zum Beispiel «Welche Wirkungen stellen Sie fest? Was hat sich seit Einführung der Massnahmen verändert? Woran erkennen Sie das?», bei den unterschiedlichen Interessengruppen relativ leicht zu ermitteln sind. Mit einem solchen Vorgehen werden zwar beabsichtigte und nicht beabsichtigte Wirkungszugeständnisse möglich, die sich jedoch nicht als Ursache-Wirkung-Zusammenhang eindeutig dem Qualitätskonzept zuordnen lassen, da die Operationalisierung der Qualität für ein solches Vorgehen keine notwendige Voraussetzung ist. Methodisch anspruchsvoller gestaltet sich jedoch die Wirkungsmodellierung, die klare Vorstellungen bezüglich des Qualitätsgegenstandes, seiner Wirkungsabsichten und Wirkungsmassnahmen verlangt, damit diese in einem logischen Szenario abgebildet werden können.

Unabhängig davon, ob die Evaluation von Qualitätskonzepten in entwicklungsfördernder formativer oder bilanzierender summativer Absicht geplant wird, sich einer internen oder externen Perspektive verschreibt, als Selbst- oder Fremdevaluation angelegt ist, ist es zentral, hervorzuheben, dass die Evaluation eine stark steuernde Funktion übernimmt. Die Evaluation trägt eine grosse Verantwortung für eine transparente Bewertung und Validierung eines - wenn von Qualität die Rede ist - insgesamt wenig transparenten Sachverhalts und leistet mit einem professionellen, standardbasierten Vorgehen einen wichtigen Beitrag zu einer realistischen Einschätzung von Wirkungen. Jedoch droht auch der Evaluation mit einem solchen Vorgehen letztlich die Gefahr einer verkürzten Sichtweise, nämlich dann, wenn im bildungspolitisch dominierten Qualitätsdiskurs innerhalb kurzer Zeit Wirkungsnachweise zu Legitimationszwecken verlangt werden. 


\section{Fallbeispiel: Nationales Projekt "Zertifizierung von berufsbildenden Schulen" zur Einführung von Qualitätsmanagementsystemen auf der Grundlage von ISO 9001 an berufsbildenden Schulen}

Mit dem folgenden Beispiel soll aufgezeigt werden, dass die Validität und damit verbunden die mögliche Wirksamkeit des Qualitätskonzepts nicht alleine durch das Konzept selbst, sondern im pädagogischen Diskurs durch Wirkungsattributionen expliziter wie impliziter Natur hergestellt sowie mit der Evaluation teilweise verworfen werden.

Im Zusammenhang mit dem vom Bundesamt für Berufsbildung und Technologie BBT 1995 initiierten und 2003 abgeschlossenen Impulsprojekt "Zertifizierung von berufsbildenden Schulen» 5 begann in der Schweiz die - wenn auch äusserst bescheidene - pädagogische Rezeption der Tauglichkeit von teilweise aus der Industrie stammenden Qualitätskonzepten für Bildungsinstitutionen. Diesbezügliche Meilensteine sind sowohl die Analysen von Gonon, Hügli, Landwehr, Ricka und Steiner (1999) als auch die Arbeit von Hügli (2000). Während sich Gonon et al. (1999) mit den meisten zu dieser Zeit verfügbaren und denkbaren Qualitätskonzepten, darunter auch mit der Norm ISO 9001 von 1994, befasst haben, hat Hügli (2000) versucht, die Norm ISO 9001 auf Bildungsinstitution zu übertragen und sie damit für Bildungsinstitutionen zu interpretieren. Kennzeichnend ist, dass sich beide Analysen jeweils mit der Norm ISO 9001 auseinandergesetzt haben, die 1994 erschien und dass die sogenannte «neue», vollständig überarbeitet Normenserie, die 2000 publiziert wurde, nicht Gegenstand der Rezeption war. ${ }^{6}$ Dennoch kann dieser auf der alten Norm basierenden Rezeption eine diskursive Wirkung beigemessen werden, die bis heute den pädagogischen Diskurs im Zusammenhang mit Darstellungen der Norm ISO 9001 massgebend beeinflusst. ${ }^{7}$ Weiter ist festzuhalten, dass weder die kontinuierliche Weiterentwicklung der Norm ISO 9001 seit 1994 bis heute noch die eingehende Befassung mit der «neuen» Normenserie (ISO 9000:2000) Gegenstand einer pädagogischen Auseinandersetzung waren, wie dies Gonon et al. (1999) noch für die «alte» Norm getan haben. Nachfolgend werden mit Bezug auf die in Kapitel 2 aufgeführten Qualitätskategorien die Hauptannahmen aufgegriffen, wie sie von Gonon et al. (1999) diskutiert wurden. Die Diskussion der Norm ISO 9001:1994 verdeutlicht, dass ihr kaum Wirkungen auf der Ebene des Unterrichts beigemessen und etliche, wenn auch in der Analyse nicht eindeutig belegte Kritikpunkte dargelegt werden. Zu diesen zählen eine «Bürokratisierung», die durch die «Regelungen des Qualitätsmanagementhandbuchs» gefördert wird, die «die Qualität nicht verbessern, sondern Innovationen verhindern und die Selbstverantwortung [...] einschränken»(Gonon et al., 1999, S. 21). Damit wird implizit angedeutet, dass ein Qualitätsmanagementsystem auf dieser Grundlage nicht einem Qualitätsverständnis im Sinne eines pädagogischen, dynamischen, entwicklungsfördernden und transformatorischen Ansatzes genügen 
kann (siehe Kp. 3). Dargelegt wird dies auch mit der Aussage, wonach die Norm «nur einen unbedeutenden Einfluss auf den Unterricht» (Gonon et al., 1999, S. 22) hat und nicht «die Verbesserung der inhaltlichen Qualität der Bildungsarbeit», sondern "die Klärung der Organisation» im Mittelpunkt steht. In Bezug auf die Rechenschaftslegung gegenüber Dritten wird der Norm ISO 9001 eine mögliche Wirkung mittels Zertifikat zugesprochen, denn die Schule «kann möglicherweise [...] glaubhafter machen, dass sie sich um Qualität bemüht» und vielleicht hat das Zertifikat auch eine "marketingstrategische Bedeutung» (Gonon et al., 1999, S. 22). Während der erste Teil der Aussage auf ein Qualitätsverständnis verweist, das die Verantwortlichkeit der Bildungsinstitution betont, einen adäquaten Gegenwert zu liefern oder sich zumindest dafür einzusetzen, deutet der zweite Teil der Aussage auf ein funktionales, zweckorientiertes Qualitätsverständnis, hier auf die Orientierung am Kunden hin (Kap. 3). Dies wird auch durch die aufgegriffene Qualitätsdefinition bestätigt, die Qualität als die vom Kunden vereinbarte oder erwartete Qualität definiert (Gonon et al., 1999, S. 20). Mit der Betonung, dass die Norm als mögliches Hilfsmittel zur Analyse und Steuerung der Management- und Verwaltungsprozesse eingesetzt werden kann (Gonon et al., 1999, S. 21), wird zusätzlich ein Qualitätsverständnis im Sinne der fehlerfreien Realisierung vordefinierter Anforderungen bei gleichzeitigem Erfüllen von Minimalstandards angesprochen (Kap. 3).

Mit den dargelegten Ausführungen wird deutlich, dass die Rezeption stark normativ geprägt ist und sich den unterschiedlichen Qualitätskategorien zuordnen lässt. Damit wird einerseits die Vielschichtigkeit der Argumentation im Zusammenhang mit Wirkungszuschreibungen an die Norm ISO 9001 herausgehoben. Andererseits kann mit diesem Beispiel exemplarisch aufgezeigt werden, dass die pädagogische Diskussion zwar gewisse Wirkungszugeständnisse organisatorischer und administrativer Art macht, sobald aber die "pädagogische» Qualitätskategorie der Transformation und der Ermächtigung angesprochen wird, dezidiert keine Wirkungszusprüche zulässt. Ein anderes Bild zeigen jedoch die Wirkungseinschätzungen mittels Evaluation des Projekts, die im Folgenden kurz dargelegt werden.

Das BBT bezieht im Abschlussbericht über das Projekt (Nationaler Rapport, Zertifizierung von berufsbildenden Schulen, 2004) zu den erreichten Wirkungen bilanzierend Stellung. Die am Projekt beteiligten Schulen wurden aufgefordert, sich u.a. zum Thema «erzielte Resultate» zu äussern. Im Sinne von «subjektiven Wirkungseinschätzungen» werden folgende positive Ergebnisse festgestellt: Das Qualitätsmanagementsystem wird als wichtiger integraler Bestandteil des Schulalltags aufgefasst, das die Basis für das Handeln und Denken bildet. Es bietet inhaltliche Kontinuität, die über ein Schulführungshandbuch gewährleistet wird. Durch klare Regelung von Abläufen erhöhen sich die Freiräume und der nach Prozessen gestaltete Schulalltag bietet fast nur Vorteile. Mit dem Qualitätsmanagementsystem wurde zur Auseinandersetzung mit der eigenen Qualität und dem Profil angeregt, was auch das Selbstverständnis der Schulen verändert 
hat, die nun zu eigentlichen Kompetenzzentren werden. Darüber hinaus hat sich die Schulkultur erheblich verbessert, und das Qualitätsmanagementsystem erlaubt eine schnelle Integration neuer Mitarbeitenden. Die Monitoringinstrumente (Selbst- und Fremdevaluation, neue Feedbackkultur) werden als hilfreich für die Weiterentwicklung angesehen. Durch klare Regelung der Abläufe bleibt mehr Zeit für das Kerngeschäft, den Unterricht sowie für andere Projekte. Sogar auf Unterrichtsebene werden Wirkungen attribuiert, nämlich, dass die neue Feedbackkultur dazu beigetragen hat, die Klassentüren zu öffnen und teilweise direkte Auswirkungen auf didaktische und methodische Aspekte hat (Nationaler Rapport, Zertifizierung von berufsbildenden Schulen, 2004, S. 21f.).

Diese durchaus positive bildungspolitische Bilanzierung verdeutlicht, dass dem Projekt und der Norm ISO 9001 für eine Bildungsinstitution auf allen Ebenen, d.h. auf Führungs- und organisatorischer als auch auf Ebene des Unterrichts relevante Wirkungen beigemessen werden. Die Norm ISO 9001 wird daher - und damit stehen die beschriebenen Wirkungen im Gegensatz zur pädagogischen Rezeption - durchaus als Basis für ein Qualitätsmanagementsystem aufgefasst, die im Sinne der pädagogischen Qualitätskategorie den transformatorischen und qualitativen Wandel der Aufgabe der Bildungsinstitution positiv beeinflussen kann. Allerdings kann an dieser Stelle mit einer methodischen Sichtweise gefragt werden, inwieweit die Wirkungseinschätzungen, losgelöst vom je spezifischen Kontext der Bildungsorganisationen, verallgemeinert werden können, zumal die beschriebenen Wirkungen subjektiven Bewertungen unterliegen. Darüber hinaus ist zu fragen, ob mit Hilfe einer Wirkungsmodellierung die gleichen Wirkungen aufgezeigt werden können (Kap. 4). Diese Frage ist insofern berechtigt, als mit dargelegten Ergebnissen aufgezeigt werden kann, dass die Wirkungen sehr allgemein formuliert, nicht weiter spezifiziert und im Sinne eines eher allgemeinen Verständnisses dem Qualitätsmanagementsystem zugeschrieben werden (Kap. 2).

Losgelöst von den methodischen Einwänden kann bilanzierend festgehalten werden, dass kontextuelle Faktoren sowohl die pädagogische Rezeption als auch die bildungspolitische Evaluation beeinflussen. Während für die pädagogische Diskussion die kritische Haltung gegenüber der Wirksamkeit eines Qualitätskonzepts aus der Industrie hervorzuheben ist, deutet die äusserst positive Bilanzierung der Evaluation auf die bildungspolitische Rechenschaftslegung hin.

\section{Fazit}

Die in diesem Artikel angesprochene Problematik im Zusammenhang mit der Bestimmung und Evaluation von Qualität verdeutlicht, dass Qualität und Evaluation ihre Wirkung erst im spezifischen Kontext mit entsprechend subjektiv zu erbringender Bewertungsleistung entfalten. Nur durch Offenlegung und Diskussion der impliziten Bewertungskriterien können sowohl die Konzepte als 
auch die Evaluation nutzbringend verwendet werden. Wird dies unterlassen, droht die Gefahr, dass Qualität und Evaluation als zentrale Steuerelemente eher in einer allgemeinen, wenig konkreten nutzenorientierten Absicht verwendet werden und damit an einem programmatischen, nicht weiter spezifizierten Diskurs haften bleiben.

\section{Anmerkungen}

1 Der Begriff Qualitätskonzepte versteht sich hier als Sammelkategorie, die jegliche Qualitätsbemühungen im Bildungssystem umfassen, wozu genauso Qualitätsmanagementsysteme, Programme mit einer intendierten (Qualitäts-)Absicht oder allgemeine Massnahmen zählen, die Qualität zum Thema haben.

2 Ergänzend hierzu ist zu bemerken, dass sich die Schulleistungsforschung vornehmlich mit pädagogisch-psychologischen Konzepten befasst, die einen individuellen und interaktionistischen Fokus (Schüler, Eltern, Lehrer) verfolgen und auf der Mikroebene, d.h. auf Unterrichtsebene angesiedelt sind.

3 Aus den dargelegten Gründen favorisieren zum Beispiel Fend (1998) und Dubs (2003) eine Qualitätskonzeption, die mehrere Ebenen der Bildungsorganisation mit einbezieht und den Unterricht als zentrale Grösse in den Mittelpunkt stellt. Auch Ditton (2000) sieht in der klaren Fokussierung der Unterrichtsqualität die Legitimation für Qualitätsansätze.

4 Gemäss der ISO 9000er Normenserie bezeichnen inhärente Merkmale stabile und ständige Eigenschaften zur Erfüllung der Anforderungen (ISO 9000: 2005 Normenserie, S. 13).

5 Das BBT hat mit diesem Projekt den Aufbau von Qualitätsmanagementsystemen an berufsbildenden Schulen auf der Grundlage der ISO 9000er Normenserie gefördert und auch finanziell unterstützt. Gemäss Abschlussbericht (2004) haben über hundert Berufsschulen in der achtjährigen Laufzeit des Projekts ein Zertifikat erworben.

6 Mit Publikation der Analysen von Gonon et al. ist eine Auseinandersetzung mit der neuen Normenserie auch kaum möglich, da die Analyse vor Publikation dieser fertiggestellt wurde.

7 So beziehen sich beispielsweise die Darstellungen der ISO 9000er Normenserie von Büeler (2004, S. 282) explizit auf Gonon et al., und Dubs (2003, S. 42) verweist in seiner Auseinandersetzung auf Hügli (2000) und orientiert sich zur Charakterisierung der ISO Normenserie für Schulen an Posch und Altrichter (1997). Altrichter und Posch (1997) beziehen sich in ihrer Analyse zwar auf die ISO 9000er Normenserie, berufen sich jedoch auf den so genannten British Standard BS 5750 von 1987, den sie als auf die Norm ISO 9001 übertragbar ansehen (S. 57 ff.).

\section{Literatur}

Altrichter, H. \& Posch, P. (1997). Möglichkeiten und Grenzen der Qualitätsevaluation und Qualitätsentwicklung im Schulwesen. Innsbruck: StudienVerlag.

Bellmann, J. (2006). Bildungsforschung und Bildungspolitik im Zeitalter «Neuer Steuerung».Zeitschrift für Pädagogik, 52, 487-504.

Beywl, W. (2004). Das A-B-C der wirkungsorientierten Evaluation. Glossar. Köln: UnivationInstitut für Evaluation Dr. Beywl \& Associates GmbH.

Büeler, X. (2004). Qualitätsevaluation und Schulentwicklung. In R. Stockmann (Hrsg.), Evaluationsforschung. Grundlagen und ausgewählte Forschungsfelder (S. 259-286). Opladen: Leske+Budrich. 2. Auflage.

Criblez. L. \& Osterwalder, F. (2004). Editorial zum Thema bildungspolitische Analysen. Schweizerische Zeitschrift für Bildungswissenschaften, 26, 5-9. 
Ditton, H. (2000). Qualitätskontrolle und Qualitätssicherung in Schule und Unterricht. Ein Überblick zum Stand der empirischen Forschung. Zeitschrift für Pädagogik, 41. Beiheft, 73-93.

Dubs, R. (2003). Qualitätsmanagement für Schulen. Studien und Berichte des IWP, Band 13. St. Gallen: Institut für Wirtschaftspädagogik.

Eidgenössisches Volkswirtschaftsdepartement (EVD), Bundesamt für Berufsbildung und Technologie (BBT) (Hrsg.). (2004). Nationaler Rapport. Zertifizierung von berufsbildenden Schulen. Bern: (s.n.).

Fend, H. (1998). Qualität im Bildungswesen. Schulforschung zu Systembedingungen, Schulprofilen und Lehrerleistung. Weinheim; München: Juventa.

Gonon, Ph., Hügli, E., Landwehr, N., Ricka, R. \& Steiner, P. (1999). Qualitätssysteme auf dem Prüfstand. Die neue Qualitätsdiskussion in Schule und Bildung - Analyse und Perspektiven. Aarau: Sauerländer.

Harvey, L. \& Green, D. (2000). Qualität definieren. Fünf unterschiedliche Ansätze. Zeitschrift für Pädagogik, 41. Beiheft, 17-39.

Heid, H. (2000). Qualität. Überlegungen zur Begründung einer pädagogischen Beurteilungskategorie. Zeitschrift für Pädagogik, 41. Beiheft, 41-51.

Helmke, A. \& Weinert, F.E. (1997). Bedingungsfaktoren schulischer Leistung. In F.E. Weinert (Hrsg.), Psychologie des Unterrichts und der Schule. Enzyklopädie der Psychologie, D, Serie Pädagogische Psychologie, Bd. 3, (S. 71-176). Göttingen: Hogrefe.

Hesse, H.-G. \& Wottawa, H. (1997). Methodische Probleme der Unterrichtsforschung. In F.E. Weinert (Hrsg.), Psychologie des Unterrichts und der Schule. Enzyklopädie der Psychologie, D, Serie Pädagogische Psychologie, Bd. 3, (S. 37-69). Göttingen: Hogrefe.

Hügli, E. (2000). Die ISO-Norm für Schulen. Interpretation und Anwendungshilfe zur ISONorm 9001, speziell für berufsbildende Schulen. Aarau: Sauerländer.

Joint Committee on Standards for Educational Evaluation. Sanders, J. R. (Hrsg.) (2000). Handbuch der Evaluationsstandards. Opladen: Leske + Budrich.

Kromrey, H. (2004). Qualität und Evaluation im System Hochschule. In R. Stockmann (Hrsg.), Evaluationsforschung. Grundlagen und ausgewählte Forschungsfelder (S. 233-258). Opladen: Leske+Budrich.

Landwehr, N. \& Steiner, P. (2003). Qualität durch Evaluation und Entwicklung. Konzepte, Verfahren und Instrumente zum Aufbau eines Qualitätsmanagements an Schulen. Bern: h.e.p.Verlag.

Lersch, R. (2004). Schule als Sozialsystem: Theoretische Modellierungsvarianten und ihr Potenzial für Analyse und Entwicklung dieses pädagogischen Feldes. In W. Böttcher \& E. Terhart (Hrsg.), Organisationstheorie in pädagogischen Feldern (S. 71-84). Wiesbaden: VS Verlag für Sozialwissenschaften.

Maritz, B., Gerber, N., Perrottet, A., Rüegg, A., Studer, H., Wenger, B. \& Winkelmann, F. (2006). Bewertungsbuch für Schulen. Eine Anleitung zur Evaluation der Schulqualität auf der Grundlage des Modells der European Foundation for Quality Management (EFQM) . Bern: h.e.p.-Verlag.

Scheerens, J. \& Bosker, R. J. (1997). The Foundations of Educational Effectiveness. Elsevier: (s.n.).

Schweizerische Normen-Vereinigung (SNV) (Hrsg.) (2000). Schweizer Norm (ISO 9001: 2000), Qualitätsmanagementsysteme - Anforderungen. Winterthur: (s.n.).

Schweizerische Normen-Vereinigung (SNV) (Hrsg.) (2005). Schweizer Norm (ISO 9000: 2000), Qualitätsmanagementsysteme - Grundlagen und Begriffe. Winterthur: (s.n.).

Stockmann, R. (2002). Qualitätsmanagement und Evaluation - Konkurrierende oder sich ergänzende Konzepte? Zeitschrift für Evaluation, 2, 209-243.

Widmer, T., Landert, Ch. \& Bachmann, N. (2000). Evaluations-Standards der Schweizerischen Evaluationsgesellschaft (SEVAL-Standards). www.seval.ch 
Schlagworte: Qualitätsmessung, Methoden der Qualitätsmessung, Effektivität der Qualitätsmessung, Evaluation des Bildungssystems

\section{Qualité et évaluation: effets implicites et défis méthodologiques}

\section{Résumé}

L'article met l'accent sur la tension normative entre qualité et évaluation du point de vue des visées de pilotage et des exigences de mesures d'efficacité de la politique éducative. Le critère de qualité du système éducatif revêt actuellement une fonction prescriptive extrêmement importante tant pour le système de l'enseignement que pour chacune des institutions scolaires. Comme catégorie visant le but et le programme éducatifs, la qualité s'appuie sur un fondement normatif qui n'est pas explicité sur le plan conceptuel général et qui est inhérent aux concepts de qualité. À titre d'exemple, sur la base du projet national «certification des écoles pour la formation professionnelle», il est démontré qu'une compréhension peu explicite du concept de qualité entraîne des évaluations non spécifiques influencées par des facteurs implicites et dépendant du contexte. Ce faisant, les renseignements fournis n'informent que de manière limitée sur les mécanismes profonds à l'œuvre et ne permettent pas d'esquisser des solutions possibles.

Mots clés: mesures de qualité, méthodes de mesures, efficacité des mesures de qualité, évaluation des systèmes éducatifs

\section{Qualità e valutazione: effetti impliciti e sfide metodologiche}

\section{Riassunto}

L'articolo pone l'accento sul conflitto normativo tra qualità e valutazione alla base delle misure di controllo politico-educative e delle esigenze metodologiche di performance. La qualità del sistema educativo assume, nella sua attuale accentuazione, la funzione di una categoria prescrittiva estremamente importante sia per il settore dell'insegnamento che per le singole istituzioni scolastiche. In veste di categoria specifica e programmatica, la qualità poggia su una base normativa che, in un primo momento, non viene illustrata sul piano concettuale generale e verte sui concetti di qualità. Con l'esempio del progetto nazionale «certificazione delle scuole per la formazione professionale», si evidenzia come una comprensione poco esplicita del concetto di qualità sia alla base di valutazioni delle performance gestite in maniera non specifica, come siano controllate da fattori impliciti e immanenti a livello contestuale e pertanto possano fornire soltanto delle nozioni di scarsa rilevanza sulle dinamiche più intrinseche e sulle possibili soluzioni. 
Parole chiave: misura della qualità, metodi di misura, efficacia delle misure di qualità, valutazione dei sistemi educativi.

\section{Effects of Implicit Norms on Quality Assessment - a Methodological Challenge}

\section{Summary}

The article focuses on the normative conflict between quality and assessment at the basis of measures of political-educational control and methodological demands of performance. In its current accentuation, the quality of the educational system assumes the function of an extremely important prescriptive category, both for the teaching sector and for single scholastic institutions. As a specific and programmatic category, quality rests on a normative basis which, at first, is not explicated at the general conceptual level and regards the concepts of quality. The example of the national project «certification of schools for vocational training» shows, how poor understanding of the quality concept inevitably leads to quite unspecific performance assessments, as they are controlled by implicit and immanent factors at the contextual level and thus can only provide notions of scant relevance about the most intrinsic dynamics and possible solutions.

Keywords: quality assessment, methods of performance assessments, implicit norms, educational policy, efficacy of quality assessments, evaluation of educational system 